\title{
Eosinophilic leukaemia in association with a double Philadelphia chromosome
}

\author{
G. STOCKDILL \\ M.R.C.P.
}

S. E. HARTLEY*

B.Sc., Ph.D.

\author{
N. C. Allan \\ F.R.C.P., F.R.C.Path. \\ Department of Haematology, and ${ }^{*} M R C$ Clinical and Population Cytogenetics Unit, \\ Western General Hospital, Edinburgh
}

\begin{abstract}
Summary
A case of eosinophilic leukaemia in association with chromosomal abnormalities including a double Philadelphia chromosome is reported. Comment is also made on the cardiological problems which arise in this condition.

\section{Introduction}

Eosinophilic leukaemia (EL) is a rare disorder. It is associated with a marked peripheral blood and marrow eosinophilia and almost invariably with myocardial involvement, frequently leading to cardiac failure. A close affinity with chronic granulo-
\end{abstract}

cytic leukaemia (CGL) has been suggested and although cytogenetic abnormalities have been $\delta$ recorded in only a limited number of patients, $7 \AA$ have had a Philadelphia chromosome ( $\mathrm{Ph}^{\prime}$ positive). N A case of $\mathrm{EL}$ with a double $\mathrm{Ph}^{\prime}$ chromosome and $\infty$ an abnormal chromosome 12 is now reported.

\section{Case report}

A 59-year-old male presented with a 3-month history of excessive tiredness and weight loss of $14 \overrightarrow{0}$ $\mathrm{kg}$ over one year. Physical examination reveal marked hepato-splenomegaly. Investigations: $10.0 \mathrm{~g} / \mathrm{dl}$; WCC $27.2 \times 10^{\circ} / 1$ (neutrophils

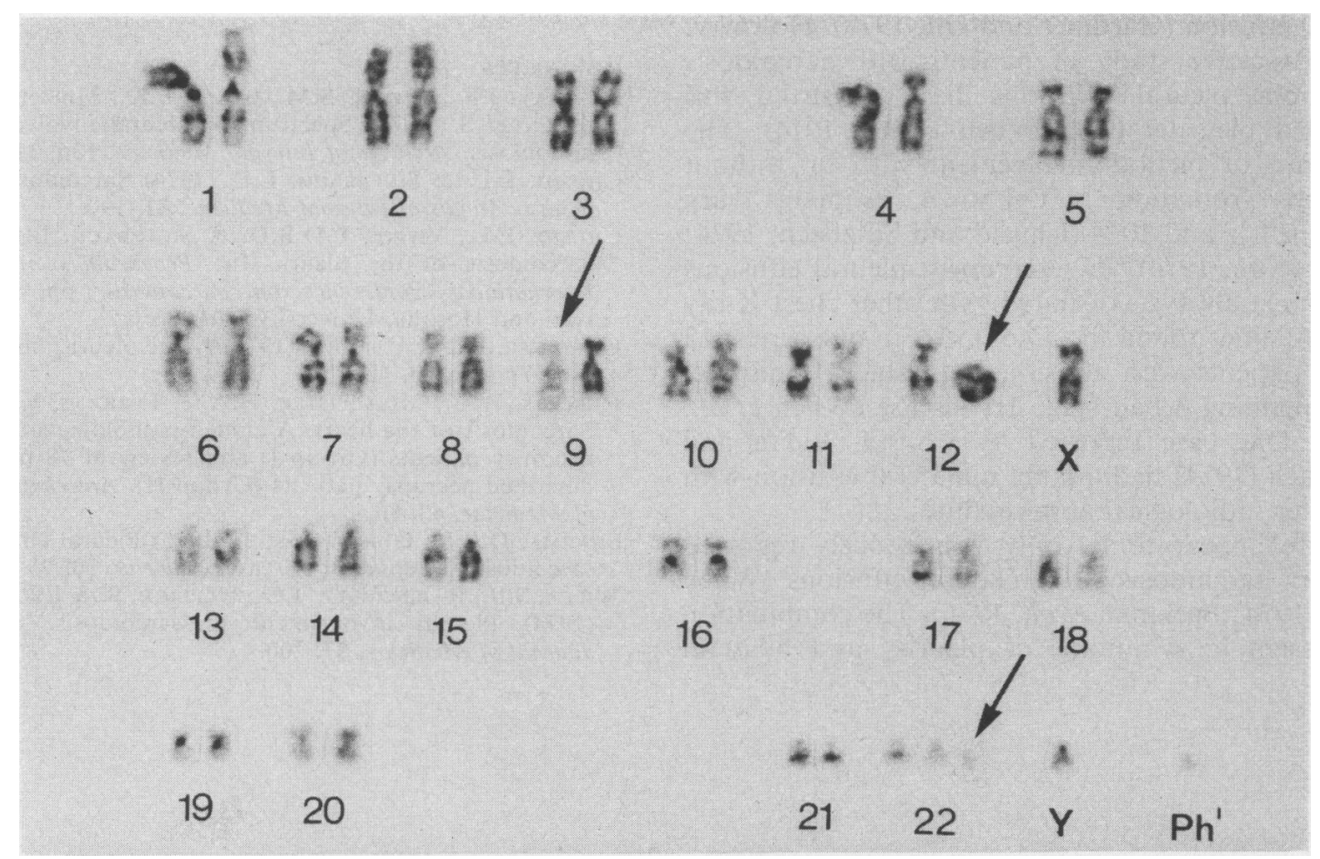

Fig. 1. Karyotype of a bone marrow cell following Trypsin-Giemsa banding: $48, X Y, t(9 ; 22)(q 34 ; q 11)$, r(12) (p12q22), $+\operatorname{de1}(22)(\mathrm{q} 11),+22$. 
lymphocytes $7 \%$, monocytes $6 \%$, eosinophils $78 \%$, basophils $1 \%$, myelocytes $1 \%$ ); platelets $30 \times 10 \% 1$. Blood film revealed many large eosinophils with large sparsely scattered granules, hypersegmented nuclei and cytoplasmic vacuolation. Marrow aspirate was hypercellular with $80 \%$ cells being eosinophilic in origin but no increase in blast cells, the predominant cell being the eosinophilic myelocyte. Antinuclear factor and rheumatoid factor were both negative, as were chest X-ray and ECG. The echocardiograph, however, showed slight thickening of the left ventricular endocardium. Immunoglobulins including IgE were normal. Neutrophil alkaline phosphatase (NAP) score was normal. Eosinophil function studies showed normal $F_{c}$ and $C_{3}$ receptors, which responded normally to enhancement by chemotactic factors.

Chromosome analysis on a marrow aspirate showed $2 \mathbf{P h}^{\prime}$ chromosomes, one resulting from a classical 9;22 translocation and one derived from the 22q- translocation chromosome. In addition, one of the no. 12 chromosomes was in a ring form and an extra chromosome 22 was present, giving a karyotype of $48, \mathrm{XY}, \mathrm{t} \quad(9 ; 22) \quad(\mathrm{q} 34 ; \mathrm{q} 11), \quad \mathrm{r}(12)$ (p12 q22), $+\operatorname{de1}(22)$ (q11), +22 (Fig. 1).

In view of the possibility of this 'mature cell' EL being a variant of CGL, treatment was started with a combination of 6-mercaptopurine $50 \mathrm{mg}$ daily, busulphan $2 \mathrm{mg}$ daily and allopurinol $300 \mathrm{mg}$ daily. This resulted in a rapid reduction in the eosinophil count, eventually down to normal levels, the absolute neutrophil count being relatively unaffected (Fig. 2).
Hepatosplenomegaly regressed but unfortunately, as the eosinophil count was falling, the patient developed left ventricular failure (A on Fig. 2) which proved extremely slow to respond to therapy. Chemotherapy was discontinued at this point but had to be re-instituted some time later after development of a haemorrhagic pleural effusion and rising eosinophil count (B on Fig. 2). Repeat chromosome analysis on bone marrow after $\mathbf{3}$ months' therapy showed reversion to a completely normal male karyotype 46,XY. Chemotherapy in a reduced dosage maintained a normal eosinophil count for 12 weeks, but the patient then entered an accelerated phase with a rising eosinophil count and increasing splenomegaly which proved refractory to further treatment, and he required considerable support in terms of blood and platelet transfusions. He developed lobar pneumonia and cardiac failure preterminally, and died 11 months after presentation.

Post-mortem examination confirmed the presence of widespread leukaemic deposits in bone, lymph nodes, spleen and muscle. The heart showed left ventricular dilatation with extensive endomyocardial fibrosis (Fig. 3) and thrombotic vegetations overlying this fibrosis and on the mitral valve. Leukaemic deposits were also noted in the myocardium of the left ventricle.

Discussion

It is now accepted that EL exists as a distinct entity and represents the more aggressive end of the spectrum of disease covered by the term 'hypereosinophilic syndrome' (Hardy and Anderson, 1968). It has been classified into 3 morphological categories

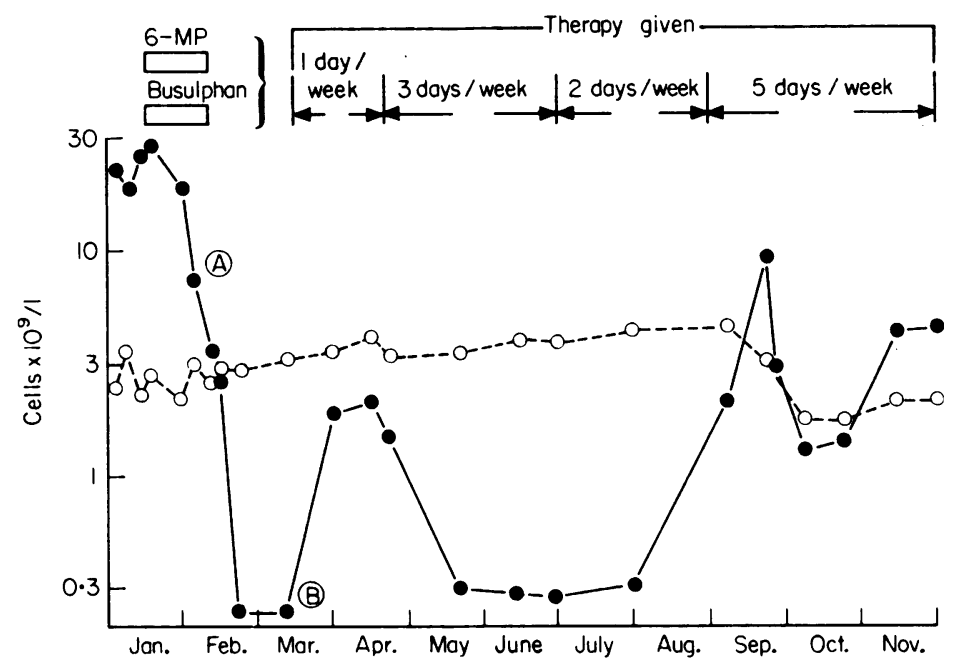

FIG. 2. Haematological response to chemotherapy. $0_{---0}$ neutrophils; eosinophils. 


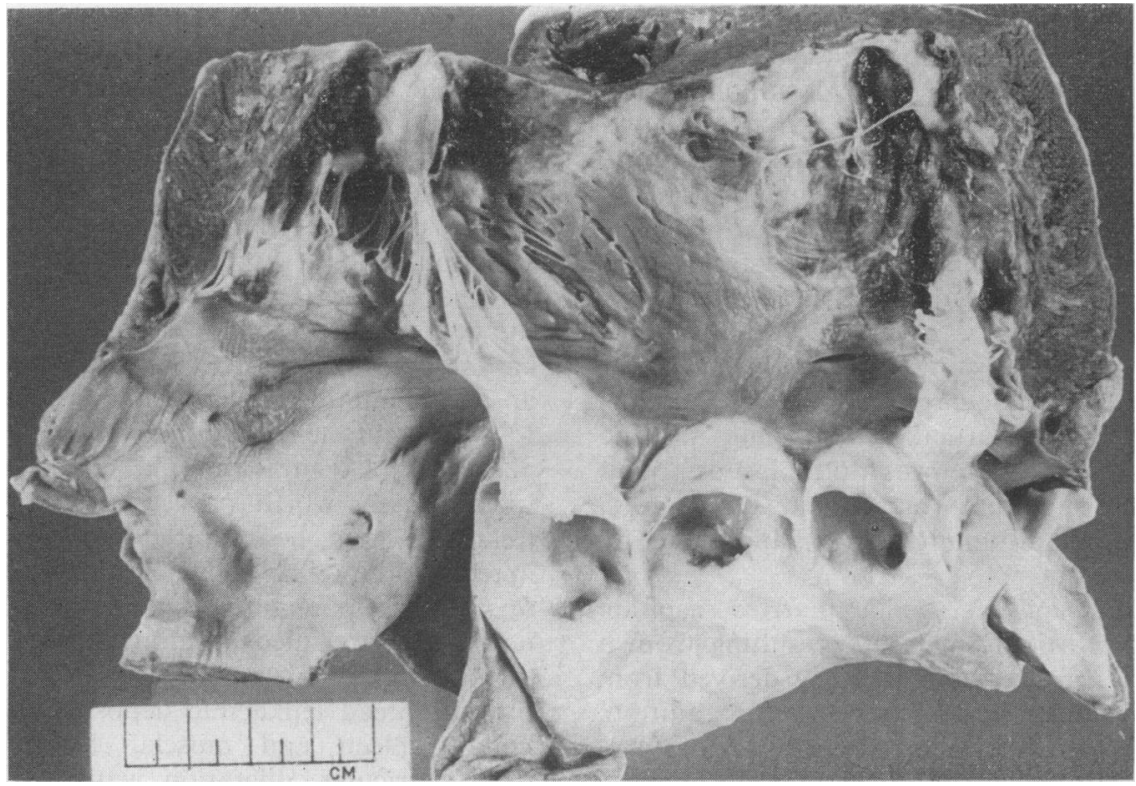

Fig. 3. Heart showing extensive endomyocardial fibrosis.

-acute blastic, immature, and mature (Benvenisti and Ultman, 1969). The present authors consider that this case falls into the 'mature' category. Although cytogenetic studies have been performed on only a limited number of patients with EL, abnormalities have been frequently uncovered (Weinfeld, Westin and Swolin, 1977). Seven cases have been found with $\mathrm{Ph}^{\prime}$ chromosome and this has led to the suggestion that EL is a variant of CGL and that EL could be similarly classified into $\mathrm{PH}^{\prime}$ negative and $\mathrm{Ph}^{\prime}$ positive groups. This patient had a double $\mathrm{Ph}^{\prime}$ chromosome-a feature frequently found in transforming CGL, and occasionally at the time of diagnosis (International Workshop on Chromosomes in Leukaemia, 1978). In addition, he had an abnormal $\mathrm{C}$ group chromosome -12 . $\mathrm{C}$ group chromosome abnormalities are a frequent finding in addition to the $\mathrm{Ph}^{\prime}$ in classical CGL, and imply a poorer prognosis (Sakurai, Hayata and Sandberg, 1976). This may also be the case in EL. However, the normal NAP score, normal neutrophil count and severe cardio-respiratory problems encountered in this patient are features not usually associated with CGL. Similarly, the striking effect of therapy on the eosinophils with sparing of neutrophils supports the claim of existence of separate precursor cells for both eosinophils and neutrophils. The response to this combination therapy recently shown to be effective in CGL (Allan, Duvall and Stockdill, 1978) was initially good but the normal karyotype encountered during the 'remission' phase with loss of $\mathrm{Ph}^{\prime}$ positivity wh unexpected as it is an extremely uncommon occur . rence in classical CGL. This suggests that in th: case the chromosome abnormalities were confine to the eosinophil precursors.

Endomyocardial fibrosis, a feature associated with hypereosinophilia in other diseases (Chusid et al., $\stackrel{\mathbb{\perp}}{\unrhd}$ 1975) and problems of cardiac failure are known $\vec{\overrightarrow{ }}$ to occur in eosinophilic leukaemia. The rapid lysis $\frac{3}{3}$ of eosinophils achieved by treatment in this case $\bar{P}$ may have precipitated the severe cardiac failure $\overrightarrow{0}$ encountered, and emphasizes the necessity for care- $-\overrightarrow{0}$ ful reduction of the hypereosinophilia in such cases.

\section{References}

Allan, N.C., Duvall, E. \& Stockdill, G. (1978) Combination chemotherapy for chronic granulocytic leukaemia. Lancet, ii, 523.

Benvenisti, D.S. \& Ultman, T.E. (1969) Eosinophilic leukemia. Annals of Internal Medicine, 71, 731.

Chusid, M.J., Dale, D.C., West, B.C. \& WolfF, S.M. (1975) The hypereosinophilic syndrome. Medicine. Baltimore, $54,1$.

HaRdY, W.R. \& ANDERSON, R.E. (1968) The hypereosinophilic syndromes. Annals of Internal Medicine, 68, 1120.

INTERNATIONAL WORKSHOP ON CHROMOSOMES IN LEUKAEMIA N (1978) Proceeding of the 1st International Workshop on $\sigma$ Chromosomes in Leukaemia, Helsinki, August 1977. British Journal of Haematology, 39, 305, 311.

Sakurai, M., Hayata, I. \& Sandberg, A.A. (1976) Prognostic value of chromosomal findings in $\mathrm{Ph}^{\prime}$ positive chronic myeloid leukaemia. Cancer Research, 36, 313.

Weinfeld, A., Westin, J. \& Swolin, B. (1977) $\mathrm{Ph}^{\prime}$ negative eosinophilic leukaemia with trisomy 8 . Scandinavian Journal of Haematology, 18, 413. 